\title{
Sublytic C5b-9 Induces Glomerular Mesangial Cell Apoptosis Through miR- 3546/SOX4/Survivin Axis in Rat Thy-1 Nephritis
}

\author{
Chunyan Yao Fengxia He $^{b}$ Longfei Liua Zhiwei Zhanga Chenhui Zhao \\ Wen Qiu $^{\mathrm{a}}$ Dan Zhao Jing Zhang ${ }^{\mathrm{a}}$ Mengxiao Xie ${ }^{\mathrm{a}}$ Yajuan Gong ${ }^{\mathrm{a}}$ \\ Tianyi Yu ${ }^{a}$ Lu Xia ${ }^{a}$ Baomei Qian ${ }^{a}$ Yingwei Wang ${ }^{a}$
}

${ }^{a}$ Department of Immunology, Nanjing Medical University, Nanjing, bepartment of Pathology, Second Affiliated Hospital of Nanjing Medical University, Nanjing, 'Department of Medicine, First Affiliated Hospital of Nanjing Medical University, Nanjing, China

\section{Key Words}

Thy-1 Nephritis • Sublytic C5b-9 • Glomerular mesangial cells • Apoptosis • miR-3546 • SOX4 - Survivin

\begin{abstract}
Background/Aims: The activation of complement system and the formation of C5b-9 complex have been confirmed in the glomeruli of patients with mesangioproliferative glomerulonephritis (MsPGN). However, the role and mechanism of C5b-9-induced injury in glomerular mesangial cell (GMC) are poorly understood. Rat Thy- $1 \mathrm{~N}$ is an animal model for studying MsPGN. It has been revealed that the attack of $C 5 b-9$ to the GMC in rat Thy- $1 N$ is sublytic, and sublytic $C 5 b-9$ can cause GMC apoptosis, but the underlying mechanism is not fully elucidated. To explore the role and regulatory mechanism of C $5 b-9$ in MsPGN lesion, we used rat Thy- $1 \mathrm{~N}$ model and first detected the change of microRNA (miRNA) profiles both in Thy- $1 \mathrm{~N}$ rat renal tissues (in vivo) and in the cultured GMCs with sublytic C5b-9 stimulation (in vitro). Then we determined the effect of miR-3546, which increased both in vivo and in vitro, on GMC apoptosis upon sublytic C5b-9 as well as the involved mechanism. Methods: Rat Thy-1N model was established and GMCs were treated with sublytic C5b-9. The rat renal cortex and the stimulated GMCs were obtained for miRNA microarray detection. Subsequently, the increased miRNAs were verified by real-time PCR. Meanwhile, to ascertain the ability of some miRNAs to upregulate cleaved caspase 3 and induce GMC apoptosis, the corresponding miRNA mimics were transfected into GMCs, followed by western blotting (WB) and flow cytometry mesurement. Thereafter, the miR-3546-targeted gene (SOX4) was predicted using bioinformatics approaches, and SOX4 expression in Thy- $1 \mathrm{~N}$ tissues and in the GMCs upon sublytic C5b-9 stimulation or miR-3546 mimic/inhibitor transfection were detected using real-time PCR and WB. To prove that miR-




\section{Cellular Physiology $\quad$ Cell Physiol Biochem 2018;49:1898-1917

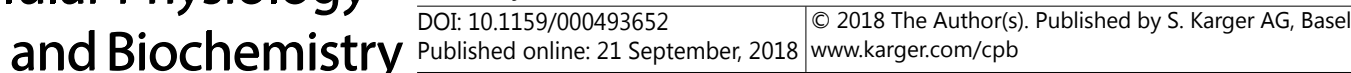 \\ Yao et al.: Sublytic C5b-9 Induces GMC Apoptosis via miR-3546/SOX4/Survivin}

3546 can affect SOX4 gene transcription and SOX4 can regulate survivin expression, dual luciferase reporter assay, real-time PCR, WB and chromatin immunoprecipitation (ChIP) assays were performed. Furthermore, the role of miR-3546/SOX4/survivin axis in the GMC apoptosis induced by sublytic C $5 \mathrm{~b}-9$ was examined using WB and flow cytometry. Results: Compared with normal renal tissues and untreated GMCs, there were 43 and 62 upregulated miRNAs (>2-fold) in Thy-1N tissues and sublytic C5b-9-stimulated GMCs respectively. A total of 17 miRNAs were increased both in vivo and in vitro, 11 of which were validated by real-time PCR. Among them, miR-3546 could markedly promote GMC apoptosis and inhibit SOX4 or survivin expression in response to sublytic C5b-9, and either SOX4 or survivin overexpression markedly rescued the GMC apoptosis mediated by miR-3546 mimic. Additionally, SOX4 overexpression could reverse the survivin suppression by miR-3546 mimic, and SOX4 could bind to survivin promoter (-1,278 to $-853 \mathrm{nt}$ ) and activate survivin gene transcription. Conclusion: MiR-3546/ SOX4/survivin axis has a promoting role in the GMC apoptosis triggered by sublytic C5b-9, and our findings may provide a new insight into the pathogenesis of rat Thy- $1 \mathrm{~N}$ and human MsPGN.

\section{Introduction}

Mesangioproliferative glomerulonephritis (MsPGN) is a disease with high incidence in human, such as IgA nephropathy [1]. The apoptosis and proliferation of glomerular mesangial cell (GMC) in MsPGN appear to be crucial in the subsequent increase of extracellular matrix (ECM) and the development of glomerulosclerosis [2, 3]. Although complement membrane attack complex, i.e. C5b-9, has been observed on the GMC of patients with MsPGN [4-6], the relationship between C5b-9 formation and GMC lesion of MsPGN is still unclear. Rat Thy-1 nephritis (Thy-1N) is a widely-used animal model for studying MsPGN [7-11]. Thy-1 antibody (Thy-1Ab), namely anti-thymocyte serum (ATS), administered into rats can bind to the Thy1 antigen on GMC membrane, form immune complexes (IC), and then activate complement system resulting in cell injury. It is known that the principal mediator of GMC damage in Thy- $1 \mathrm{~N}$ is C5b-9 complex [7], and our previous experiments have confirmed that although C5b-9 is found on the GMCs of Thy-1N rat, the GMCs still remain intact, indicating that the effect of C5b-9 on GMCs is sublytic [12-14]. In vitro, sublytic C5b-9 complex can be formed on GMC surface with a sublytic dose of Thy-1 Ab (namely ATS) and normal human serum $[15,16]$, and it has been documented that sublytic C5b-9 could be assembled on mouse podocytes using rabbit anti-MPC5 (immortalized mouse podocyte cells, MPC5) serum and healthy human serum [17]. Besides, our previous studies have revealed that sublytic C5b-9 stimulation can lead to GMC apoptosis, and the apoptotic GMCs are thought to be a potential contributor to the renal lesion of Thy-1N rat $[13,16,18]$.

Generally, C5b-9 complex can trigger dose-dependent signals related to target cell death and survival by membrane insertion. A study has point out that sublytic C5b-9 could induce receptor-interacting protein kinase 1 (RIPK1), receptor-interacting protein kinase 3 (RIPK3) and mixed-lineage kinase domain-like protein (MLKL) activation in cells, contributing to complement-dependent cytotoxicity [19]. Our previous research has also found that the expression of some transcription factors, e.g. activating transcription factor 3 (ATF3), early growth response gene 1 (Egr-1) and interferon regulatory factor 1 (IRF1), as well as proapoptosis molecules, e.g. growth arrest and DNA damage-inducible protein 45 (Gadd45), X-linked inhibitor of apoptosis-associated factor 1 (XAF1) and caspase 8, can significantly increase in rat GMCs attacked by sublytic C5b-9, which ultimately promote sublytic C5b-9induced GMC apoptosis $[15,16,18,20]$.

It is well accepted that protein post-translational modifications (PTMs) and micro RNA (miRNA) regulation can play an important role to target gene expression in the process of cell apoptosis [21, 22]. Our former experiments have demonstrated that GMC apoptosis evoked by sublytic C5b-9 in Thy-1N rats is not only associated with the overexpression of some transcription factors such as ATF3, IRF1, but also relevant to the upregulation of some 


\section{Cellular Physiology $\quad$ Cell Physiol Biochem 2018;49:1898-1917

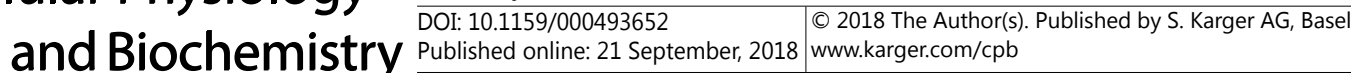 \\ Yao et al.: Sublytic C5b-9 Induces GMC Apoptosis via miR-3546/SOX4/Survivin}

transcription coregulators including histone acetyltransferase (HATs, e.g. p300) causing the acetylation of ATF3 or IRF1 modified by p300 $[15,18]$. However, the changes of miRNA expression profile both in the renal tissues of Thy-1N rats (in vivo) and in the GMCs stimulated with sublytic C5b-9 (in vitro), along with the miRNA regulatory effects on the GMC apoptosis upon sublytic C5b-9 in rat Thy-1N, remain unknown.

MiRNAs, act as endogenous small non-coding RNAs, can regulate some gene expression by binding to complementary sites in the $3^{`}$ untranslated region (3'UTR) of their target mRNAs, and promote degradation of the target mRNAs or inhibit their translation [21, 23]. Increasing evidence has proved that miRNAs can affect malignancies [24, 25], viral infection [26], diabetes [27] and IgA nephropathy [28]. Thus, the role of miRNA in the GMCs attacked by sublytic C $5 \mathrm{~b}-9$ in Thy- $1 \mathrm{~N}$ rat needs to be clarified.

In the current study, we first determined whether miRNA expression in the renal tissues of Thy-1N rat (in vivo) and in GMCs stimulated with sublytic C5b-9 (in vitro) were changed, and then which miRNAs upregulated both in vivo and in vitro were involved in sublytic C5b-9induced GMC apoptosis. Finally, the effect of miR-3546 on the apoptosis of GMCs in response to sublytic C5b-9 treatment and the embedded regulatory mechanism were further explored.

\section{Materials and Methods}

\section{Animals, cell lines and reagents}

Male Sprague-Dawley (SD) rats (180-200g) were purchased from B\&K Universal Ltd. (Shanghai, China). Rat GMC line (HBZY-1) was provided by China Center for Type Culture Collection (Wuhan, Hubei, China). HEK-293T cells were obtained from the American Tissue Culture Collection (ATCC, Manassas, VA, USA). Rabbit polyclonal anti-Thy-1 serum (Thy-1 antibody, Thy-1 Ab) was prepared in our laboratory according to previously published procedures [11, 12]. Normal human serum (NHS) from 20 healthy adult donors was used as a source of complement. Heat-inactivated serum (HIS) was obtained by incubation of NHS at $56^{\circ} \mathrm{C}$ for 30 min. Human complement C6-deficient serum (C6DS) was purchased from Sigma-Aldrich (St. Louis, MO, USA). Recombinant human C6 was obtained from Sino Biological Inc. (Beijing, China). Rat miRNA microarray was the product of Agilent Technologies (Santa Clara, CA). Lipofectamine 2000 was purchased from Invitrogen (Carlsbad, CA, USA). The vector pIRES2-EGFP was supplied by Clontech (Mountain View, CA, USA). The pGL3-promoter, pGL3-basic and pRL-SV40 luciferase reporter vectors as well as the dualluciferase reporter assay kit were purchased from Promega (Madison, WI, USA). QuickCut ${ }^{\mathrm{TM}}$ Restriction $^{\mathrm{T}}$ Endonucleases, PrimeSTAR Max DNA Polymerase and PrimeScript ${ }^{\mathrm{TM}} \mathrm{RT}$ reagent kit were purchased from TaKaRa (Shiga, Japan). Bulge-Loop ${ }^{\text {TM }}$ RT primers and miRNA qRT-PCR primers were designed and produced by RiboBio (Guangzhou, China). SYBR ${ }^{\circledast}$ Green Master Mix and HiScript ${ }^{\circledR}$ II qRT SuperMix were purchased from Vazyme (Nanjing, China). Monoclonal antibodies against caspase 3, survivin, SOX4 and polyclonal Ab against rat C5b-9 were provided by Santa Cruz (Santa Cruz, CA, USA). Anti- $\beta$-actin Ab was purchased from Abcam (Cambridge, UK). HRP-conjugated anti-mouse and anti-goat IgG were obtained from Cell Signaling Technology (Beverly, MA, USA). DAB HRP substrate kit was purchased from Beyotime (Shanghai, China). The chromatin immunoprecipitation (ChIP) assay kit was provided by Millipore (Bedford, MA, USA). AnnexinV Alexa Fluor 647/PI apoptosis detection kit was the product of Fcmacs (Nanjing, China).

\section{Thy-1N model establishment}

$\mathrm{SD}$ rats were divided into two groups ( $\mathrm{n}=5 \mathrm{in}$ each time point/group), namely Thy- $1 \mathrm{~N}$ group and normal rabbit serum (NS) group. For Thy-1N or NS group, SD rats were given rabbit anti-Thy-1 serum containing Thy- $1 \mathrm{Ab}$ (a titer of 1:1280, $0.75 \mathrm{ml} / 100 \mathrm{~g}$ ) or the same dose of normal rabbit serum for control by intravenous injection $[11,13]$. Thereafter, rats were sacrificed and the samples of renal cortex were obtained. All animal experiments were performed in compliance with the guidelines for the care and use of laboratory animals and approved by the Institutional Animal Care and Use Committee of Nanjing Medical University. 


\section{Cellular Physiology Cell Physiol Biochem 2018;49:1898-1917 \begin{tabular}{l|l|l} 
and Biochemistry Published online: 21 September, 2018 & $\begin{array}{l}\text { (c) } 2018 \text { The Author(s). Published by S. Karger AG, Basel } \\
\text { www.karger.com/cpb }\end{array}$ \\
\hline
\end{tabular}}

\section{GMCs culture and sublytic C5b-9 determination}

Rat GMCs were cultured in modified Eagle's medium (MEM) supplemented with $10 \%$ fetal bovine serum (FBS) as previously described $[13,15]$. Rabbit Thy- $1 \mathrm{Ab}$ and normal human sera (NHS, provide heterologous complement) were used to assemble C5b-9 on rat GMC membrane to avoid homologous restriction factors such as CD59 [29], which can disrupt C5b-9 formation on the cell membrane [10, 30, 31]. To ensure that C5b-9 attack was insufficient to lead to cell lysis, lactate dehydrogenase (LDH) was detected in the supernatant of cultured GMCs and $<0.5 \%$ LDH release was regarded as a sublytic effect $[13,32,33]$. Based on a checkerboard titration test, the suitable concentration of Thy- $1 \mathrm{Ab}$ and NHS used in this study was $5 \%$ Thy- $1 \mathrm{Ab}$ and $3 \%$ NHS respectively. To prove that the effect on GMCs was indeed due to sublytic C5b-9 stimulation, GMCs were treated with 5\% Thy- $1 \mathrm{Ab}+3 \%$ NHS (namely sublytic C5b-9 group), and 5 control groups, namely 5\% Thy-1 Ab, 5\% Thy-1 Ab + 3\% HIS, 5\% Thy-1 Ab + 3\% C6DS, 5\% Thy-1 Ab + 3\% C6DS + C6 (2 mg/L) and MEM $[15,16]$.

\section{Immunohistochemical examination}

Freshly frozen sections $(4 \mu \mathrm{m})$ of the rat renal tissues were examined by immunohistochemical staining to determine the $\mathrm{C} 5 \mathrm{~b}-9$ formation in rat glomeruli (at 10 minutes after Thy- $1 \mathrm{Ab}$ or normal rabbit serum injection). All sections were immersed with 5\% normal rabbit serum (1:20) for 30 min to reduce non-specific background staining, and then incubated with goat anti-rat C5b-9 polyclonal Ab (diluted 1:200), followed by HRP-conjugated secondary Ab incubation. The sections were visualized using a DAB HRP substrate kit and observed directly under light microscopy (LM).

\section{miRNA arrays}

The changes in miRNA expression profiles in the renal tissues of Thy-1N rat (in vivo) and GMCs stimulated by sublytic C5b-9 (in vitro) were determined using the rat miRNA microarray, which contain probes for 719 microRNAs from miRBase database (Release 19.0). The rat miRNA microarray assay and data analysis were performed at Shanghai Biotechnology Corporation according to the protocols of the Agilent miRNA microarray system. Briefly, miRNAs molecules in total RNA extracted from the renal tissues of Thy-1N rats or the GMCs stimulated by sublytic C5b-9 were labeled, and then each slide was hybridized with 100ng Cy3-labeled RNA using miRNA complete labeling and Hyb kit according to the manufacturer's instructions. The agilent microarray scanner G2565BA and feature extraction software 10.7 were used to scan the slides with default settings. Raw data were normalized by quantile algorithm, gene spring software 11.0 .

\section{MiRNA mimics or miR-3546 inhibitor synthesis and transfection}

The mimics of miR-21-3p, miR-134-5p, miR-150-3p, miR-202-3p, miR-212-3p, miR-298-5p, miR328a-5p, miR-409-3p, miR-3546, miR-3564, miR-3584-5p, negative control and FAM labeled negative control as well as miR-3546 inhibitor, inhibitor negative control and FAM labeled inhibitor negative control were purchased from GenePharma (Shanghai, China). These miRNA mimics and miR-3546 inhibitor were transfected by lipofectamine 2000 reagent, and the transfection efficiency was examined by the fluorescence of FAM. Three different concentrations ( $40 \mathrm{nM}, 80 \mathrm{nM}$ and $160 \mathrm{nM}$ ) were used in dose-dependent experiment of miRNA mimics. The final concentrations were $80 \mathrm{nM}$ and $160 \mathrm{nM}$ for miRNA mimics and miR-3546 inhibitor, separately.

\section{Plasmids construction and transfection}

To construct the plasmids of wild type (WT) and mutant (mut) SOX4 3'UTR, the selected region of SOX4 3'UTR including position 1072-1078 (target region of miR-3546) was amplified and inserted into the Xbal site of the pGL3-promoter vector. The mut SOX4 3'UTR was constructed by General Biosystems, Inc

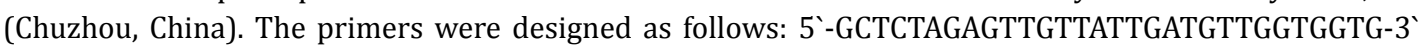
(forward) and 5`-GCTCTAGAAGATGGAGCGTCCGTGAG-3` (reverse).

Meanwhile, the plasmids of rat SOX4 and survivin were constructed. The coding fragment of SOX4 or survivin was amplified using the primers

(pIRES2/SOX4, forward 5'-CGGAATTCGAGACTCTAGGTTGGCGG-3` and reverse

5'-CGGGATCCTCTCCTTTCTCCTTCCACTCTCCA-3'; 


\section{Cellular Physiology Cell Physiol Biochem 2018;49:1898-1917 \begin{tabular}{l|l|l|l|l|l}
\hline DOI: 10.1159/000493652 2018 The Author(s). Published by S. Karger AG, Basel & and Biochemistry
\end{tabular}

pIRES2/survivin, forward $55^{`}$-CGGAATTCTCATGGGTGCTACGGC-3` and reverse 5`-CGGGATCCTAGGAGGACTCATCAGAAGG-3`)

from normal rat complementary DNA (cDNA) and inserted into the EcoRI/BamHI sites of the pIRES2-EGFP vector. Besides, rat SOX4 shRNA plasmid pGpU6/GFP/Neo-SOX4 (shSOX4-1, 5`-GGGCTCTTCGTCGTCCGATGA-3’,shSOX4-2, 5`-GCCAAGAGGCAGGAGAGGAGA-3`), and survivin shRNA plasmid pGpU6/GFP/Neo-suvivin (5'-CCGAGAATGAGCCTGATTTTT-3') as well as scrambled control shRNA (shCON) were also constructed by GenePharma. These plasmids were transfected into GMCs by lipofectamine 2000. The transfection efficiency was determined by green fluorescence protein (GFP) or corresponding proteins.

Additionally, the plasmids of pGL3/survivin full-length promoter (FL, -4, 300 to -1nt) and four truncated promoters $(-4,160$ to $-1 \mathrm{nt},-3,180$ to $-1 \mathrm{nt},-2,620$ to $-1 \mathrm{nt}$, and $-2,130$ to $-1 \mathrm{nt})$ were constructed by inserting rat survivin promoter fragments into the SmaI/XhoI sites of the pGL3-basic vector. The primers were designed as follows:

FL, 5`TCCCCCGGGCACATTTGACCTGGGCCAG-3`(forward); -4, 160 to -1nt,

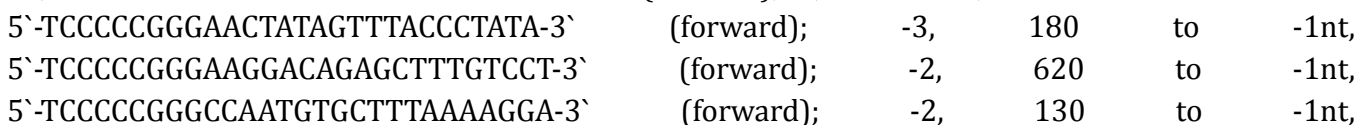

5`TCCCCCGGGCTAGCCTTTGACCAGTTGA-3`(forward)

All reverse primers were 5`-CCGCTCGAGAGCGTCGCCACAATCCCTAATT-3`.

\section{Quantitative real-time PCR}

Total RNA was extracted from the rat renal tissues and GMCs using TRIzol reagent. For miRNA or mRNA detection, $1 \mu \mathrm{g}$ RNA was reverse transcribed into cDNA using PrimeScript ${ }^{\mathrm{TM}}$ RT reagent kit and specific Bulge-Loop ${ }^{\mathrm{TM}}$ RT primers or HiScript ${ }^{\circledR}$ II qRT SuperMix respectively. Then, quantitative real-time PCR (qPCR) was performed using specific Bulge-Loop ${ }^{\mathrm{TM}}$ miRNA qRT-PCR primers or specific primers and $\mathrm{SYBR}^{\circledR}$ Green Master Mix in a Bio Rad CFX96 system. The primers were designed as follows:

SOX4, 5 `-GAAGATGACCTGCTCGACCT-3`(forward) 5`-GTGCAATAGTCCGGGAACTC-3` (reverse); survivin, 5 `-CCTTTGCTGGGCAACCTGGA-3`(forward) 5`-GCCTTGCACAACCCTCCCTT-3`(reverse);

$\beta$-actin, 5`-TCACCCACACTGTGCCCATCTATGA-3`(forward) 5`-CATCGGAACCGCTCATTGCCGATAG-3` (reverse).

All samples were normalized to internal control U6 or $\beta$-actin. Fold changes were calculated with the $2^{-\Delta \Delta} \mathrm{Ct}$ method.

Western blotting (WB)

Rat renal tissues and GMCs were lysed using RIPA lysis buffer, followed by BCA protein quantification. Extracted protein samples ( $40 \mu \mathrm{g}$ per lane) were separated using 12\% sodium dodecyl sulfate polyacrylamide gel electrophoresis (SDS-PAGE) and then transferred onto polyvinylidene fluoride (PVDF) membrane. After blocking with 5\% non-fat milk in TBS-T buffer for $1 \mathrm{~h}$, the membranes were incubated with anti-caspase 3 (dilution 1:200), anti-SOX4 (1:200), anti-survivin (1:200) and anti- $\beta$-actin $1: 1000)$ respectively. Washed with TBS-T, the membranes were further incubated with HRP-conjugated anti-mouse IgG (1:10000) for 1 $\mathrm{h}$. The bands were visualized by regular X-ray film using an ECL detection system. The density of the bands was analyzed using Quantity One software (Bio-Rad).

Rescue assays

PIRES2/SOX4, pIRES2/survivin or pIRES2-EGFP (500ng) and miR-3546 mimic or CON mimic (80nM) were co-transfected into GMCs. At $48 \mathrm{~h}$ after transfection, the expression of SOX 4 and survivin were detected by WB, and meantime apoptotic GMC numbers were detected by flow cytometry.

Dual luciferase reporter assay

GMCs were transfected with a mixture of 500ng pGL3-promoter/WT SOX4 3'UTR or pGL3-promoter/ mut SOX4 3'UTR and 2.5ng pRL-SV40 using lipofectamine 2000. At $48 \mathrm{~h}$ after transfection, GMCs were treated or untreated with sublytic C5b-9 for $3 \mathrm{~h}$. HEK-293T cells were co-transfected for $48 \mathrm{~h}$ with a mixture of 125ng pGL3-promoter/WT SOX4 3`UTR or pGL3-promoter/mut SOX4 3`UTR, 0.625ng pRL-SV40 and 


\section{Cellular Physiology Cell Physiol Biochem 2018;49:1898-1917

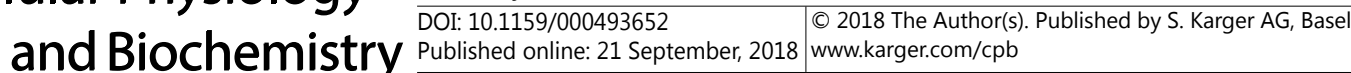 \\ Yao et al.: Sublytic C5b-9 Induces GMC Apoptosis via miR-3546/SOX4/Survivin}

40pmol miR-3546 mimic. Then, the luciferase activity of WT or mut SOX4 3'UTR reporter was measured using a dual-luciferase reporter assay kit.

For survivin promoter activity, GMCs or HEK-293T cells were transfected with corresponding plasmids. Here, GMCs transfected with 500ng FL promoter of survivin and 2.5ng pRL-SV40 for 48h were treated or untreated with sublytic C5b-9 for 3h. Additionally, GMCs or HEK-293T cells were transfected with 125ng FL or different deletion promoter reporters of survivin, $0.625 \mathrm{ng}$ pRL-SV40 and 500ng pIRES2/SOX4 for 48h. Then, the promoter activity of survivin was detected using the same method above-mentioned.

\section{ChIP assays}

ChIP assays were performed using chromatin immunoprecipitation kit. Anti-SOX4 and normal mouse IgG were used for immunoprecipitation of crosslinked protein/DNA respectively. Five regions in surivin promoter $(-2,130$ to $+1 \mathrm{nt})$ were amplified from the immunoprecipitated chromatin by PCR using corresponding primers $(-2,130$ to $-1,705 \mathrm{nt}$, forward 5 -CTAGCCTTTGACCAGTTGA-3` and reverse 5`-TAGCTATCCCAAAAGCTGTT-3`; $-1,704$ to $-1,279 \mathrm{nt}$, forward 5`-CCCGCTCGTTGTTCGGGAC-3` and reverse 5`TCCAGATGCCTGTGGTGTA-3`; $-1,278$ to -853nt, forward 5`-AGAGTCCTGTCTGAACCGG-3` and reverse $5{ }^{`}$-GGCACATCCCGAGCACAGC-3`; -852 to $-427 \mathrm{nt}$, forward 5`-CAGCCTGCTGTCTGTAGTG-3` and reverse 5 `-GGGTTTGCAGGCCCTGGCC-3`; -426 to $-1 \mathrm{nt}$, forward 5`-GAAGGAGGAAGAAACCGC-3` and reverse 5 -AGCGTCGCCACAATCCCTAATT-3`). GMCs were stimulated with sublytic C5b-9 for 3 h or transfected with pIRES2/SOX4 for $48 \mathrm{~h}$, the binding level of SOX4 on survivin promoter was detected by ChIP-qPCR assay (forward primer, 5`CTGGAGAGCTTTAGGGTAGGG-3` and reverse primer 5`-CCAGGCCACTTCACAGATTG-3`). The results were normalized to input.

\section{Flow cytometry}

Rat GMCs were transfected with miR-3546 mimic or inhibitor and corresponding plasmids as mentioned above. At $48 \mathrm{~h}$ after transfection, GMCs were treated or untreated with sublytic C5b-9 for $3 \mathrm{~h}$. Then, the GMCs $\left(1 \times 10^{5}\right)$ were resuspended in $100 \mu$ l binding buffer, incubated with Annexin V-Alexa Fluor 647 and PI for 15 min. The samples were detected on Beckman Cytoflex flow cytometer (Beckman Coulter). The percentage of apoptosis cells in a 10,000-cell cohort was analyzed by CytExpert software.

\section{Statistical analysis}

All experiments were repeated at least three times. Statistical analyses were performed using GraphPad Prism 5.0. All data are presented as means \pm SE. One-way analysis of variance (ANOVA) with Turkey's multiple comparison tests was used to determine significant differences with more than two groups. Unpaired student's t-test was used to analyze the difference between two groups. Values of $\mathrm{p}<0.05$ were considered significant.

\section{Results}

Identification of miRNAs upregulated both in the renal tissues of Thy-1N rats and in the GMCs stimulated with sublytic C5b-9

In the first place, we found C5b-9 formation on the GMCs in Thy-1N group rat but not in NS group rat at 10 minutes after Thy-1 Ab or normal rabbit serum injection (Fig. 1A). Then, to find the changes of miRNA expression both in the rat Thy- $1 \mathrm{~N}$ tissues (in vivo, compared with normal rat renal tissues) and in the GMCs exposed to sublytic C5b-9 (in vitro, compared with untreated GMCs), the miRNA array profiling (containing 719 miRNAs) was performed. The data showed that, there were 43 upregulated miRNAs and 21 downregulated miRNAs in vivo, and 62 upregulated and 31 downregulated miRNAs were found in vitro ( $>2$-fold, Fig. 1B, 1C). Specifically, as for the upregulated miRNAs, there were $25(1 \mathrm{~h}), 23(2 \mathrm{~h})$ and 37 $(3 \mathrm{~h})$ increased miRNAs in vivo (Fig. 1D), and 26 (1h), 30 (2h) and 60 (3h) in vitro (Fig. 1E). From venn diagram, we found that 17 miRNAs were in the overlap of enhanced miRNAs both in vivo and in vitro (Fig. 1B). Subsequently, the relative expression levels of these 17 miRNAs were depicted by Heatmap (Fig. 1F), and were further verified by real-time PCR. As shown in Fig. $1 \mathrm{G}$ and $1 \mathrm{H}, 11$ miRNAs among the fore-mentioned 17 miRNAs were confirmed 
Fig. 1. Analysis of the upregulated miRNAs both in rat Thy-1N tissues (in vivo) and GMCs stimulated with sublytic C5b-9 (in vitro) by miRNA microarray. (A) Representative images

immunohistochemical staining for C5b-9 in glomeruli at $10 \mathrm{~min}$ after the injection of normal rabbit serum (NS rat) or anti-Thy-1 serum (Thy$1 \mathrm{~N}$ rat). The staining of $\mathrm{C} 5 \mathrm{~b}-9$ is positive on the GMC membranes in Thy-1N rat $(\times 400)$. (B, C) The numbers of upregulated and downregulated miRNAs both in vivo and in vitro from miRNA microarray are showed by Venn diagrams. (D, E) The numbers of upregulated miRNAs both in vivo and in vitro at $1 \mathrm{~h}, 2 \mathrm{~h}$ and $3 \mathrm{~h}$ respectively from miRNA microarray. (F) Heatmap depicting relative expression levels of the 17 upregulated miRNAs both in vivo and in vitro compared with $0 \mathrm{~h}$. (G, H) The expression levels of the 17 upregulated miRNAs as detected by real-time PCR both in vivo and in vitro.

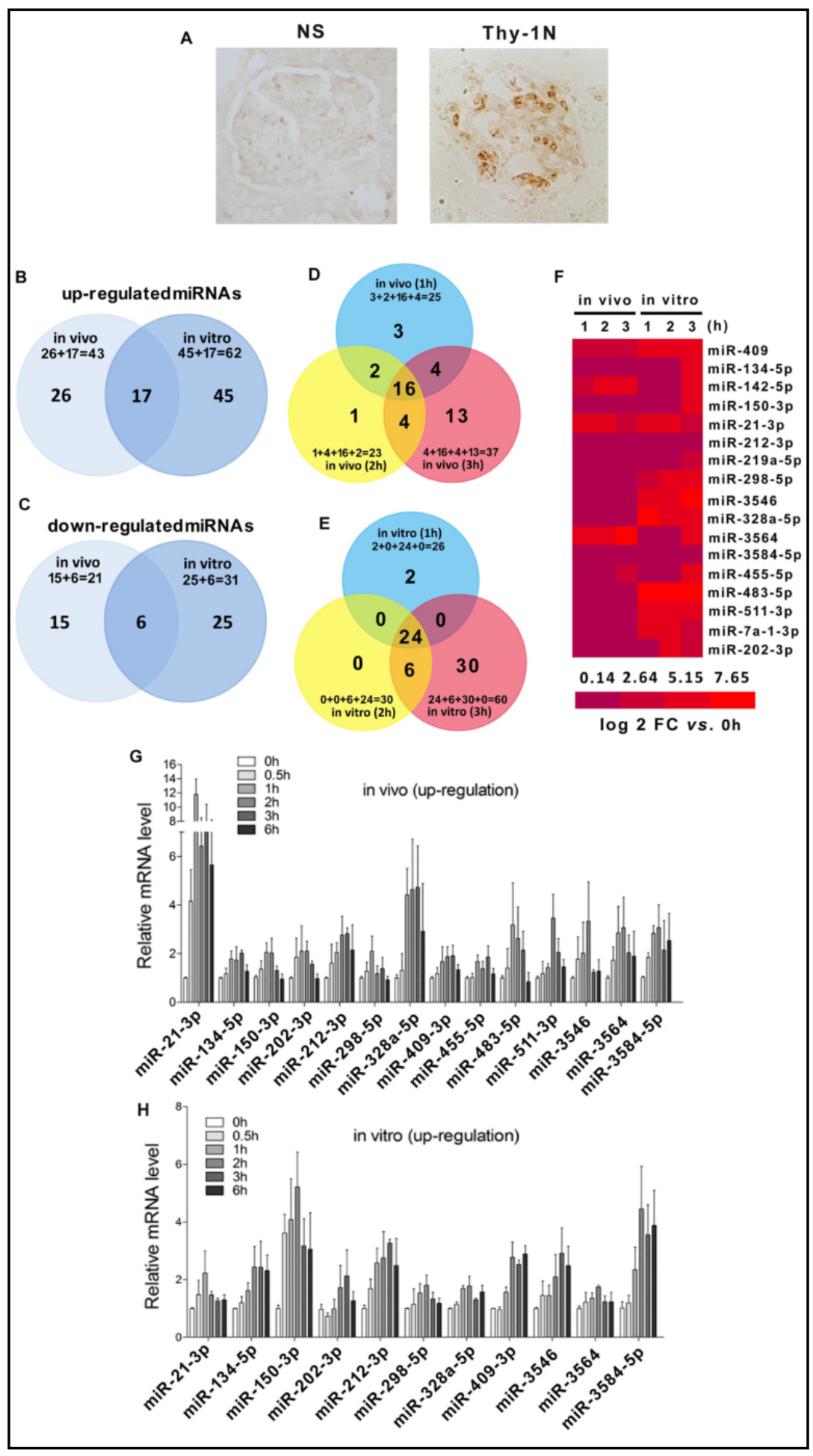




\section{Cellular Physiology $\quad$ Cell Physiol Biochem 2018;49:1898-1917

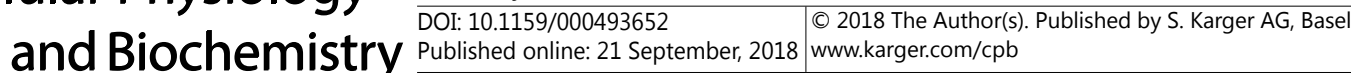 \\ Yao et al.: Sublytic C5b-9 Induces GMC Apoptosis via miR-3546/SOX4/Survivin}

as upregulated both in vivo and in vitro compared with the corresponding control groups. Almost all of these miRNAs started to increase at $0.5 \mathrm{~h}$ except for miR-202-3p and miR-409$3 p$ in vitro, the levels of 6 miRNAs (i.e. miR-150-3p, miR-202-3p, miR-511-3p, miR-3546, miR-3564 and miR-3584-5p) peaked at $2 \mathrm{~h}$ in vivo and 3 miRNAs (i.e. miR-202-3p, miR-212$3 \mathrm{p}$ and miR-3546) peaked at $3 \mathrm{~h}$ in vitro.

MiRNA-promoted cleaved caspase 3 expression and the association of miR-3546 with the GMC apoptosis upon sublytic C5b-9

To identify the GMC apoptosis-related miRNAs among the fore-mentioned 11 miRNAs increased both in vivo and in vitro, we analyzed the relationship between their expression and cleaved caspase 3 level. Firstly, the rat GMCs were transfected with the mimics of miR21-3p, miR-134-5p, miR-150-3p, miR-202-3p, miR-212-3p, miR-298-5p, miR-328a-5p, miR409-3p, miR-3546, miR-3564, and miR-3584-5p respectively, and then WB was performed to detect caspase 3 expression. The results displayed that the mimics of miR-150-3p, miR328a-5p, miR-409-3p, miR-3546 and miR-3584-5p could obviously increase the levels of cleaved caspase 3 in rat GMCs (Fig. 2A). Here, though the cleaved caspase 3 upregulation was not the most significant in the GMCs transfected with miR-3546 mimic, we found that miR-3546 mimic had the best performance in promoting the cleaved caspase 3 expression in the subsequent dose-dependent experiments (Fig. 2B). Thus, we focused on miR-3546 and further measured the change of GMC apoptosis at $48 \mathrm{~h}$ after transfection with miR3546 mimic or inhibitor following with sublytic C5b-9 treatment for $3 \mathrm{~h}$. The analysis of flow cytometry showed that the numbers of apoptotic GMCs were remarkably increased by sublytic C5b-9 stimulation and miR-3546 mimic transfection, and were notably decreased after miR-3546 inhibitor transfection following with sublytic C5b-9 incubation (Fig. 2C).

Prediction the miR-3546 target genes and detection SOX4 expression both in the renal tissue of Thy-1N rats and in the GMCs upon sublytic C5b-9

To further explore the molecular mechanism of upregulated miR-3546 promoting GMC apoptosis upon sublytic C5b-9 treatment, we used bioinformatics tools (TargetScan, miRDB) to predict putative target genes of miR-3546. The results showed that there were 1, 050 miR-3546 targeted genes in total, and further analysis by PANTHER classification system [34] showed that 73 genes were transcription factors, among which 8 genes are related to cell apoptosis (Fig. 3A). Because SOX4 was the only downregulated transcription factor both in vivo and in vitro (RT-PCR data not shown), it was selected to be further studied. The time course experiments demonstrated that along with miR-3546 level increased (peaked at $2 \mathrm{~h}$ in vivo and at $3 \mathrm{~h}$ in vitro, Fig. $1 \mathrm{~F}, 1 \mathrm{G}$ ), the expression of SOX4 was reduced concomitantly (the mRNA and protein levels minimized at $2 \mathrm{~h}$ and $3 \mathrm{~h}$ in vivo, and at $3 \mathrm{~h}$ in vitro, Fig. 3B, 3C, 3G and $3 \mathrm{H}$ ). These results suggest that expression of miR-3546 is negative correlated with SOX4 expression.

Next, to prove that the decrease of SOX 4 and the increase of miR-3546 expression in the renal tissues were induced by Thy-1N, SD rats were injected with rabbit normal serum (NS) or anti-Thy-1 serum (containing Thy-1 Ab). At $3 \mathrm{~h}$ after the nephritis initiation, the mRNA and protein of SOX4 as well as the level of miR-3546 were examined. The results showed that SOX4 expression in Thy-1N group was lower than that in NS group (Fig. 3D and 3E), and while miR-3546 level was higher in the Thy-1N group (Fig. 3F). To further confirm that the similar change in SOX4 and miR-3546 expression in rat GMCs was in fact due to sublytic C5b-9 stimulation, rat GMCs were treated with MEM, sublytic Cb-9, Thy- $1 \mathrm{Ab}$, Thy- $1 \mathrm{Ab}+\mathrm{HIS}$, Thy-1 Ab + C6DS, or Thy-1 Ab + C6DS + C6 (adding C6 back to C6DS) for $3 \mathrm{~h}$, and only sublytic C5b-9 and Thy-1 Ab + C6DS + C6 (forming sublytic C5b-9 again) remarkably decreased SOX4 expression (Fig. 3I and 3J) and notably promoted miR-3546 expression (Fig. 3K), implying that the reduction of SOX 4 and increase of miR-3546 were indeed induced by sublytic C5b-9. 


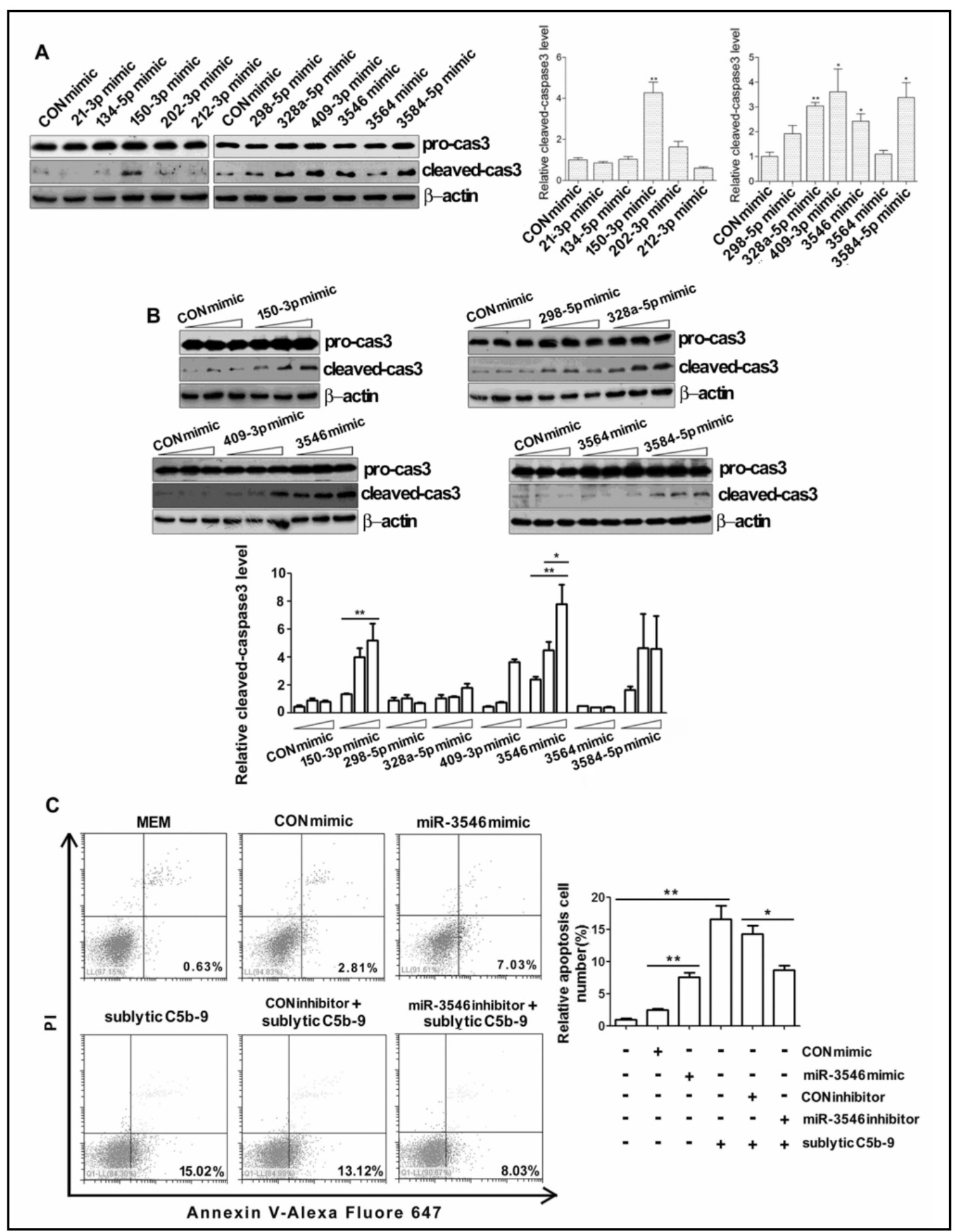

Fig. 2. Identification of the miRNAs regulating caspase 3 expression and the relationship between miR3546 and GMC apoptosis. (A) The GMCs were transfected with the corresponding miRNA mimics (80nM) and control ( $\mathrm{CON}$ ) mimic for $24 \mathrm{~h}$, and then the protein levels of pro caspase 3 and cleaved caspase 3 in the GMCs were detected using WB. (B) The indicated miRNA mimics and CON mimic were transfected into GMCs at different concentrations (40nM, 80nM and $160 \mathrm{nM})$ for $24 \mathrm{~h}$ respectively, and the protein levels of pro caspase 3 and cleaved caspase 3 in the GMCs were detected using WB. (C) GMCs were transfected with miR-3546 mimic, CON mimic, miR-3546 inhibitor and CON inhibitor respectively for 48h, followed with (or without) sublytic C5b-9 treatment for $3 \mathrm{~h}$. The numbers of apoptotic GMCs were measured by flow cytometry. Representative photographs are shown. ${ }^{*} \mathrm{P}<0.05$, ${ }^{* *} \mathrm{P}<0.01$ vs. corresponding control group.

\section{KARGER}




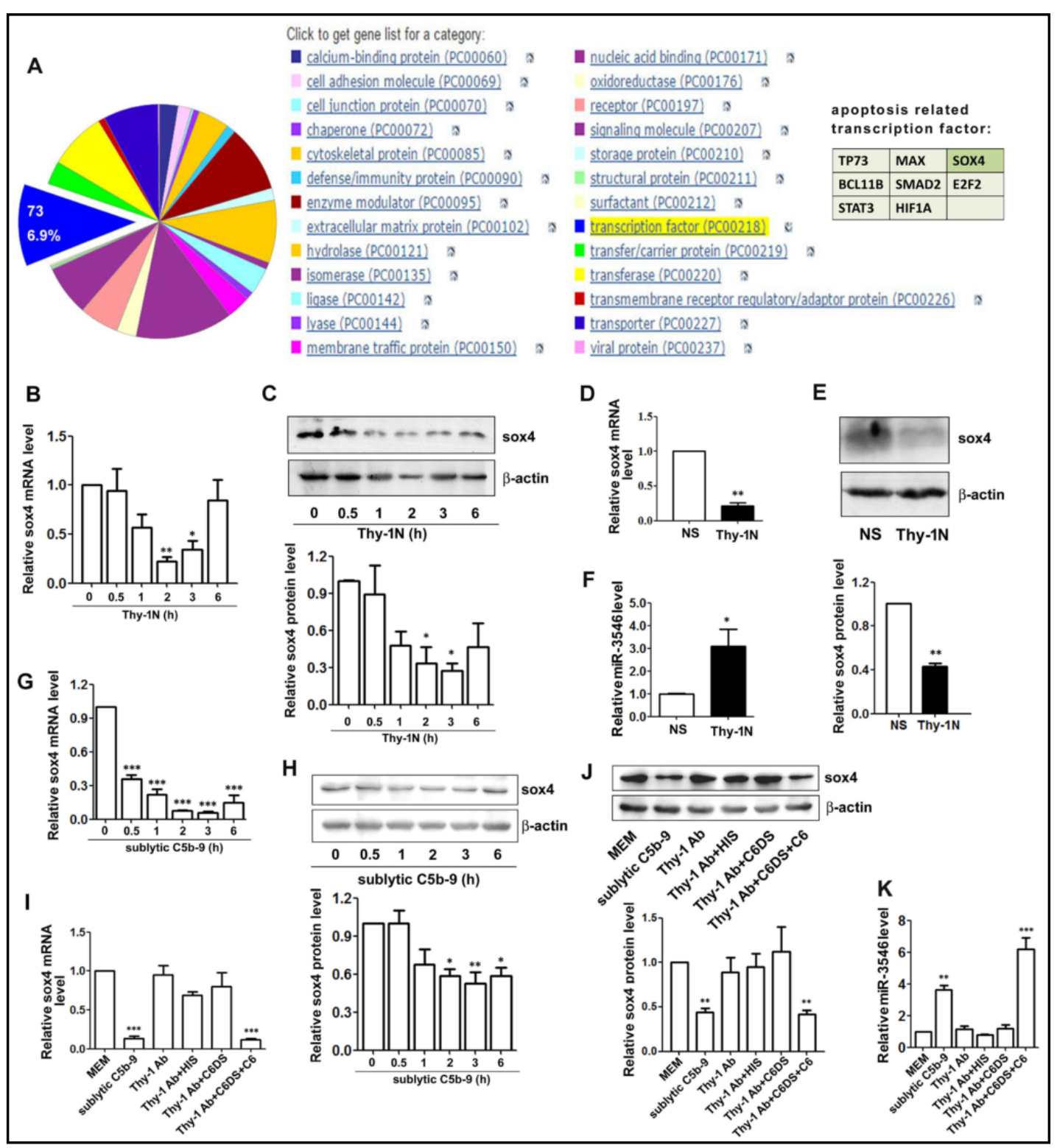

Fig. 3. Prediction of miR-3546 targeted genes and detection of the SOX4 and miR-3546 levels both in the rat Thy-1N tissues (in vivo) and in the GMCs exposed to sublytic C5b-9 (in vitro). (A) Target genes of miR3546 were predicted and analyzed by PANTHER classification system. These genes were collected from TargetScan and miRDB. (B, G) The levels of SOX4 mRNA both in vivo and in vitro at different time points were measured by real-time PCR. $(\mathrm{C}, \mathrm{H})$ The levels of SOX4 protein both in vivo and in vitro at different time points were examined using WB. (D, E, F) The levels of SOX4 mRNA and protein as well as miR-3546 mRNA at $3 \mathrm{~h}$ were detected by real-time PCR and WB in Thy-1N and NS rats. (I, J, K) The levels of SOX4 and miR3546 in the GMCs with different treatments for 3 h. Representative images are shown. ${ }^{*} \mathrm{P}<0.05,{ }^{* *} \mathrm{P}<0.01$, *** $\mathrm{P}<0.001$ vs. 0 h or NS or MEM, Thy- $1 \mathrm{Ab}$, Thy- $1 \mathrm{Ab}+\mathrm{HIS}$, Thy $-1 \mathrm{Ab}+\mathrm{C} 6 \mathrm{DS}$ groups.

Effects of miR-3546 on the expression and 3'UTR activity of SOX4 gene in the GMCS stimulated by sublytic C $5 b-9$

To determine the direct effect of miR-3546 on SOX4 expression, rat GMCs were transfected with miR-3546 mimic or inhibitor, followed by SOX4 detection. The results showed that in the GMCs transfected with miR-3546 mimic (Fig. 4A), SOX4 mRNA and protein were significantly reduced (Fig. 4B, 4C). Meantime, after GMCs were transfected with miR-3546 inhibitor followed by sublytic C5b-9 treatment for 3h (Fig. 4D), the SOX4 
expression was markedly elevated compared with CON inhibitor + sublytic C5b-9 group (Fig. $4 \mathrm{E}, 4 \mathrm{~F}$ ). Besides, to further ascertain the relationship of miR-3546 and SOX4, a luciferase reporter assay with the vectors harboring the 3'UTR of SOX4 containing either the wild type (WT) or a mut binding site for the miR-3546 was performed (Fig. 4G). The data displayed that sublytic C5b-9 could significantly suppress the WT but not mut SOX4 3'UTR activity in rat GMCs (Fig. 4H). Furthermore, in the HEK-293T cells, miR-3546 mimic also remarkably decreased the activity of WT SOX4 3'UTR, instead of mut SOX4 3'UTR (Fig. 4I). These data indicate that SOX4 is a direct target gene of miR-3546.

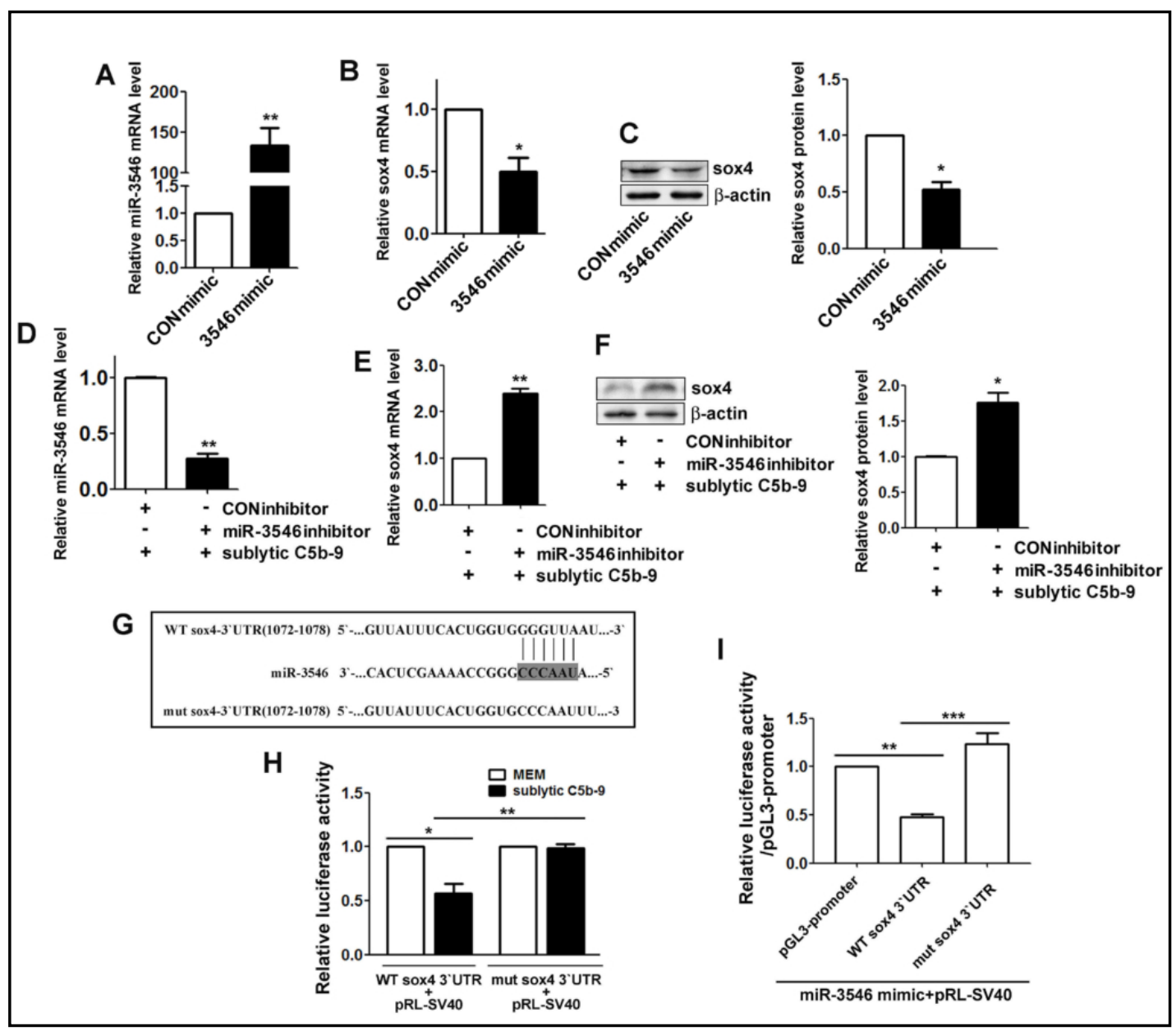

Fig. 4. Effect of miR-3546 on SOX4 gene expression and transcription in GMCs. (A-F) The GMCs transfected with CON mimic and miR-3546 mimic (80nM) respectively for $24 \mathrm{~h}$ or CON inhibitor and miR-3546 inhibitor $(160 \mathrm{nM})$ for $24 \mathrm{~h}$ followed by sublytic C5b-9 stimulation for $3 \mathrm{~h}$, and the mRNA levels of miR-3546 (A, D) or SOX4 (B, E) in the corresponding groups were detected by real-time PCR. The protein levels of SOX4 (C, F) were measured using $\mathrm{WB}$ assay. ${ }^{*} \mathrm{P}<0.05,{ }^{*} \mathrm{P}<0.01$ vs. CON mimic or CON inhibitor + sublytic C5b-9. $(\mathrm{G}, \mathrm{H})$ The GMCs were transfected with wild type (WT) or mutant (mut, the miR-3546 targeted region GGGUUA mutated to CCCAAU) of SOX4 3'UTR for 48h (G), and then exposed to sublytic C5b-9 for 3h. The activity of WT and mut SOX4 3'UTR was detected by luciferase reporter analysis (H). (I) The WT or mut 3'UTR of SOX4 was co-transfected with miR-3546 mimic into HEK-293T cells for $48 \mathrm{~h}$, and the activity of SOX4 3'UTR was also examined by reporter assay. Representative images are shown. ${ }^{*} \mathrm{P}<0.05,{ }^{* *} \mathrm{P}<0.01,{ }^{* * *} \mathrm{P}<0.001$ vs. corresponding group. 


\section{Cellular Physiology Cell Physiol Biochem 2018;49:1898-1917

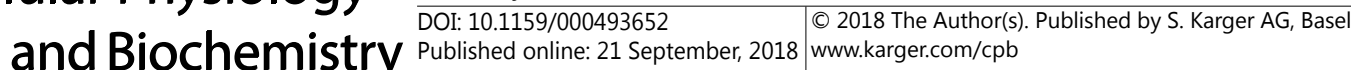 \\ Yao et al.: Sublytic C5b-9 Induces GMC Apoptosis via miR-3546/SOX4/Survivin}

Role of SOX4 and miR-3546 in regulating survivin gene expression of the GMCs treated with sublytic C $5 b-9$

It is well known that both SOX4, a transcriptional activator and survivin, an antiapoptotic molecule, can regulate cell apoptosis $[35,36]$, but whether SOX4 could regulate survivin remains unclear. Hence, we firstly detected the survivin expression both in the renal tissues of Thy- $1 \mathrm{~N}$ rats (in vivo) and in the GMCs treated with sublytic C5b-9 (in vitro). Our time course experiments showed that the survivin expression decreased markedly, reaching its lowest level at $3 \mathrm{~h}$ both in vivo and in vitro (Fig. 5A, 5C). Moreover, the level of survivin protein in Thy-1N rats significantly diminished compared with NS group (Fig. 5B). In rat GMCs treated with sublytic C5b-9 or Thy-1 Ab + C6DS + C6, the level of survivin expression was also remarkably reduced (Fig. 5D). These findings demonstrated that the decrease phase of survivin gene expression was similar to that of SOX4 gene expression, suggesting a potential relationship between survivin and SOX4. To verify this relationship in GMCs, we constructed plasmids of SOX4 overexpression and short hairpin RNA (shRNA), and utilized two shSOX4 plasmids to avoid off-target effect. Thereafter, the GMCs were transfected with the plasmids of SOX4 overexpression (pIRES2/SOX4) or shSOX4 (shSOX4-1, shSOX4-2), with or without sublytic C5b-9 stimulation for $3 \mathrm{~h}$. The results showed that SOX4 overexpression markedly enhanced survivin expression. When the cells were treated with sublytic C5b-9 for $3 \mathrm{~h}$, the survivin expression was declined together with SOX4 reduction (Fig. 5E). On the other hand, silence of SOX4 gene in the GMCs transfected with shSOX4-1 and shSOX4-2 could notably lessen the survivin expression, which was even more significant in the presence of sublytic C5b-9 attack (Fig. 5F). Besides, miR-3546 mimic or miR-3546 inhibitor (followed by sublytic C5b-9 treatment for $3 \mathrm{~h}$ ) also eminently reduced or increased survivin protein level respectively (Fig. 5G, 5H). Furthermore, pIRES2/SOX4 could rescue the survivin repression by miR-3546 mimic in GMCs (Fig. 5I). Together, these data implicate that the expression of survivin is truly regulated by SOX4 and miR-3546.

Effect of SOX4 on the survivin gene transcription and identification of binding site for SOX4 on survivin promoter

To confirm that SOX4 can directly affect survivin gene transcription, the reporter plasmids of full-length (FL) and four truncated fragments of survivin promoters, according to the SOX4 binding site prediction by JASPAR database (Fig. 6B), were constructed and transfected into GMCs or HEK-293T cells. Then, the activity of survivin promoter was measured, and the results showed that the survivin FL promoter activity was markedly suppressed by sublytic C5b-9 (Fig. 6A). Meantime, the activities of FL and 4 truncated survivin promoters in the GMCs were significantly augmented by SOX4 overexpression (Fig. 6C). Similar results were obtained in HEK-293T cells (Fig. 6D). To further determine SOX4-binding site on survivin promoter, ChIP-PCR assay was performed using five pairs of primers designed to amplify corresponding survivin promoter regions (Fig. 6E), and the results showed that the -1 , 278 to -853 nt region of survivin promoter was the effective binding site of SOX4 (Fig. 6F). Furthermore, the ChIP-qPCR results exhibited that sublyic C5b-9 stimulation or pIRES2/ SOX4 plasmid transfection markedly decreased or increased the binding level of SOX4 on the region of survivin promoter in GMCs (Fig. 6G). Collectively, these findings revealed that SOX4 could directly boost survivin gene transcription in the GMCs stimulated by sublytic C5b-9.

Effects of miR-3546/SOX4/survivin axis on sublytic C5b-9-induced cleaved caspase 3 expression and GMC apoptosis

To investigate the role of miR-3546/SOX4/survivin axis in cleaved caspase 3 expression and cell apoptosis in the GMCs triggered by sublytic C5b-9, the GMCs were transfected with the expression plasmids of SOX4 or survivin (pIRES2/SOX4, pIRES2/survivin) or shRNA (shSOX4-1, shSOX4-2, shsurvivin, with or without sublytic C5b-9 stimulation for $3 \mathrm{~h}$ ). The results showed that SOX4 overexpression markedly lowered the cleaved caspase 3 level in GMCs even though with sublytic C5b-9 incubation (Fig. 7A), it also decreased the numbers of apoptotic GMCs (Fig. 7E). Contrarily, silence of SOX4 gene remarkably elevated the 


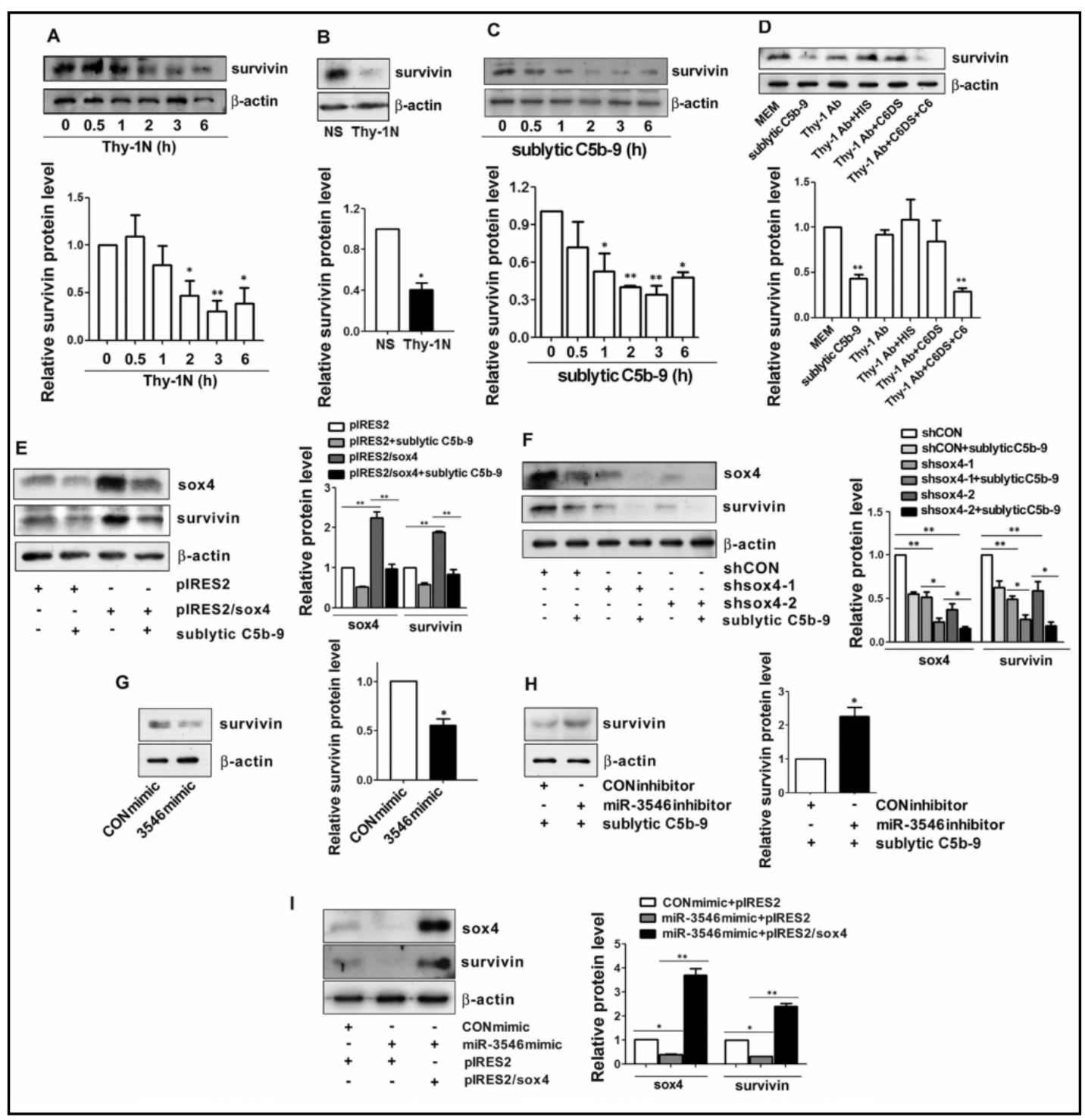

Fig. 5. The survivin expression in the rat Thy-1N tissues and GMCs upon sublytic C5b-9 stimulation as well as the regulatory role of SOX4 in survivin expression. (A, B) The protein levels of survivin in the renal tissues at different time points of Thy- $1 \mathrm{~N}$ and at $3 \mathrm{~h}$ in the Thy- $1 \mathrm{~N}$ and NS groups were measured using WB. (C, D) The survivin expression in the GMCs at different time points exposed to sublytic C5b-9 and in the GMCs with various treatments for $3 \mathrm{~h}$ was detected. ${ }^{*} \mathrm{P}<0.05,{ }^{* *} \mathrm{P}<0.01$ vs. 0 h or NS or MEM, Thy- $1 \mathrm{Ab}$, Thy$1 \mathrm{Ab}+\mathrm{HIS}$, Thy-1 Ab+C6DS. (E, F) The levels of SOX4 and survivin were measured in the GMCs transfected with pIRES2/SOX4 and shSOX4-1 or shSOX4-2 plasmids for 48h, followed with or without sublytic C5b-9 treatment for $3 \mathrm{~h}$, respectively. (G, H) The GMCs transfected with CON mimic and miR-3546 mimic (80nM) for $24 \mathrm{~h}$, or CON inhibitor and miR-3546 inhibitor (160nM) for $24 \mathrm{~h}$ with sublytic C5b-9 incubation for $3 \mathrm{~h}$, and the survivin expression was detected using WB. (I) The GMCs were co-transfected with pIRES2/SOX4 and miR-3546 mimic for 48h, and then the protein levels of SOX4 and survivin were detected using WB. Representative photographs are shown. ${ }^{*} \mathrm{P}<0.05$, ${ }^{* *} \mathrm{P}<0.01$ vs. corresponding control group. 


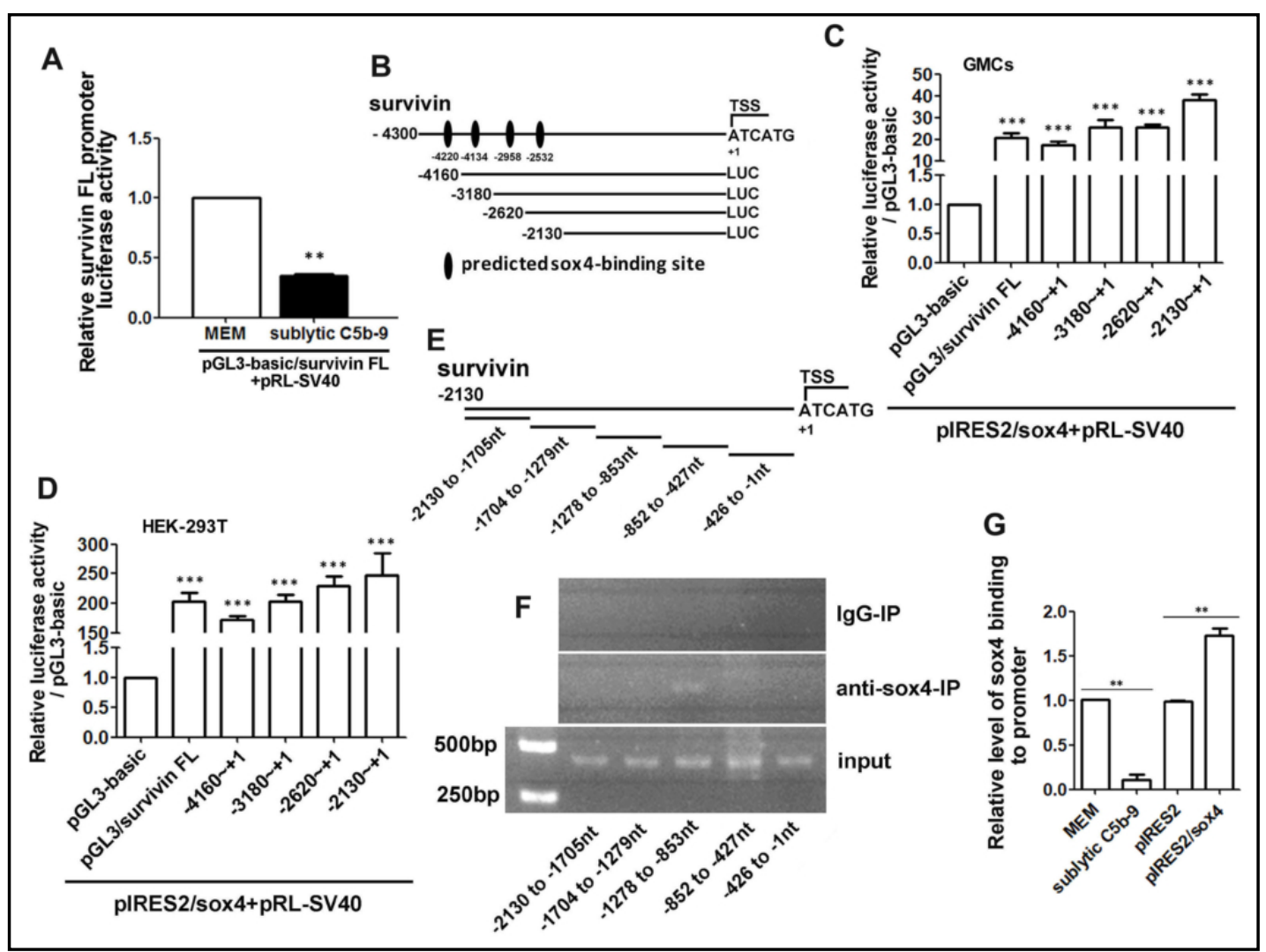

Fig. 6. The role of SOX4 in activating survivin gene promoter in the GMCs attacked by sublytic C5b-9. (A) GMCs were transfected with survivin FL promoter reporter plasmid and pRL-SV40 for 48h, followed with sublytic C5b-9 exposure for $3 \mathrm{~h}$. The FL promoter activity of survivin was detected by luciferase reporter assay. ${ }^{* *} \mathrm{P}<0.01$ vs. MEM. (B) Schematic representation of survivin promoter truncated fragments and predicted SOX4 binding sites on survivin promoter. (C, D) The pIRES2/SOX4 and pRL-SV40 plasmids were co-transfected with the above-mentioned plasmids into GMCs (C) or HEK-293T cells (D) for 48h, and then the activities of survivin FL and 4 truncated promoters were measured by luciferase reporter assay. ${ }^{* * *} \mathrm{P}<0.001$ vs. pGL3-basic. (E) Schematic diagram depicting 5 pairs of PCR primers used to amplify corresponding regions of survivin promoter (within -2,130 to $-1 \mathrm{nt}$ ). (F) ChIP assay was performed using anti-SOX4 antibody or control IgG. The immunoprecipitated DNA was purified and amplified using PCR, and the effective SOX4 response element on survivin promoter is in the $-1,278$ to -853nt region. (G) GMCs were stimulated with sublytic C5b-9 for 3h or transfected with pIRES2/SOX4 plasmid for 48h, ChIP-qPCR assay was then performed to measure the levels of SOX4 binding to the $-1,278$ to -853 nt region of survivin promoter. ${ }^{* *} \mathrm{P}<0.01$ vs. MEM or pIRES2 group.

cleaved caspase 3 expression and GMC apoptosis together with sublytic C5b-9 (Fig. 7B, 7E). Moreover, overexpressing or silencing survivin respectively inhibited or enhanced cleaved caspase 3 expression (Fig. 7C, 7D) as well as GMC apoptosis with or without sublytic C5b9 (Fig. 7E). Furthermore, pIRES2/survivin or pIRES2/SOX4 markedly rescued the GMC apoptosis mediated by miR-3546 mimic (Fig. 7F). These data suggest that the miR-3546/ SOX4/survivin axis does contribute to the sublytic C5b-9 triggered GMC apoptosis. 


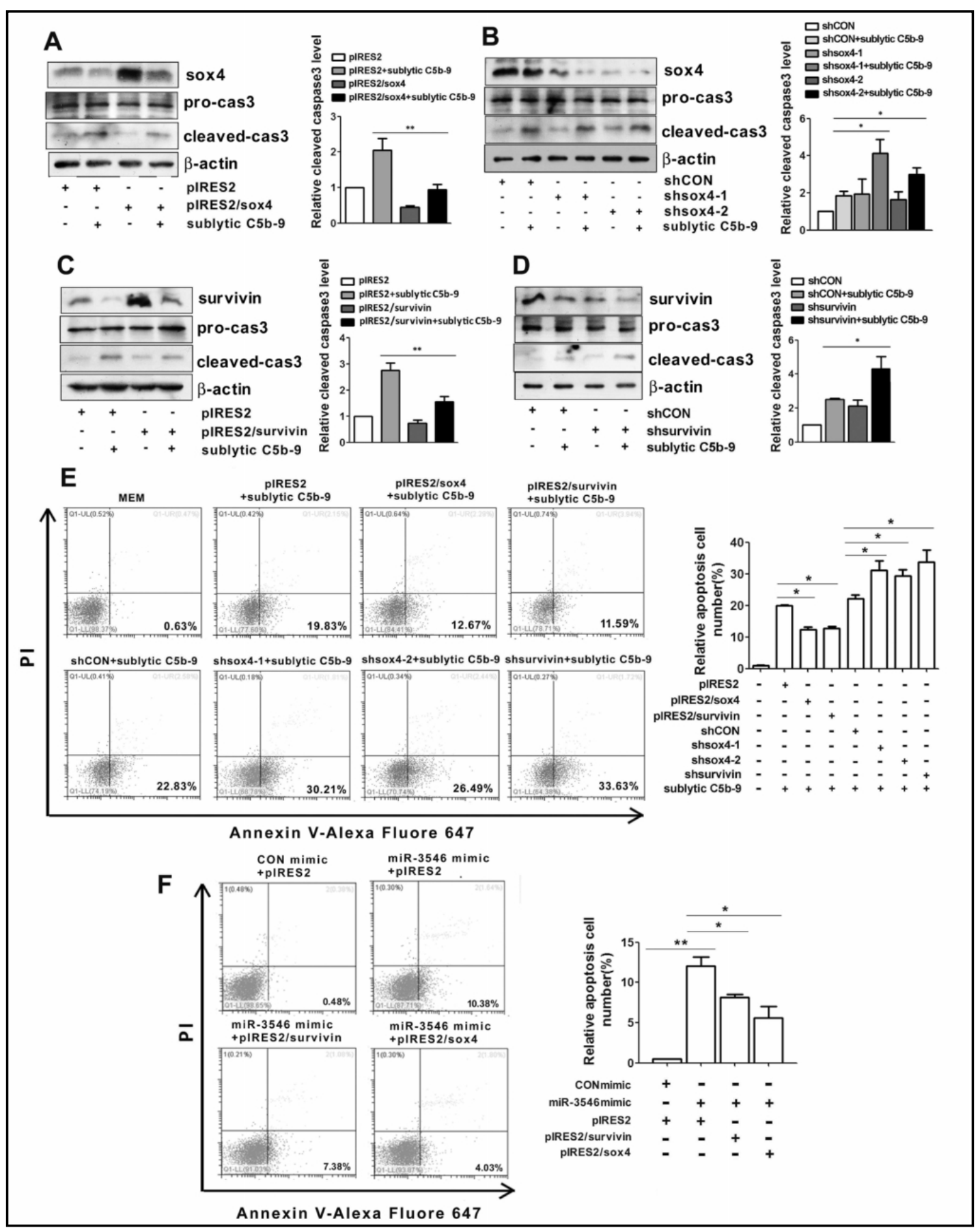

Fig. 7. The roles of SOX4 and survivin in sublytic C5b-9-induced cleaved caspase 3 expression and GMC apoptosis. (A, B) The levels of SOX4, pro caspase 3 and cleaved caspase 3 in GMCs were measured using WB at 48h after the transfection with pIRES2/SOX4 and shSOX4-1 or shSOX4-2 plasmids, followed with or without sublytic C5b-9 stimulation for $3 \mathrm{~h}$. (C, D) The levels of survivin, pro caspase 3 and cleaved caspase 3 were also detected using WB in the GMCs transfected with pIRES2/survivin and shsurvivin plasmids for 48h, with or without sublytic C5b-9 exposure for 3h. (E) GMCs were transfected with the plasmids of pIRES2/ SOX4, pIRES2/survivin, shSOX4-1, shSOX4-2 and shsurvivin respectively for 48h, and then stimulated with sublytic C5b-9 for $3 \mathrm{~h}$. The numbers of apoptotic GMCs were measured by flow cytometry. (F) GMCs were co-transfected with pIRES2/SOX4 or pIRES2/survivin and miR-3546 mimic for 48h, and then the numbers of apoptotic GMCs was detected by flow cytometry. Representative photographs are displayed. $* \mathrm{P}<0.05$, ${ }^{* *} \mathrm{P}<0.01$ vs. corresponding control group. 


\section{Cellular Physiology Cell Physiol Biochem 2018;49:1898-1917

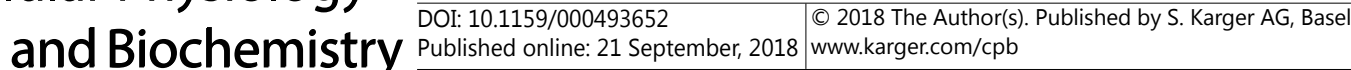 \\ Yao et al.: Sublytic C5b-9 Induces GMC Apoptosis via miR-3546/SOX4/Survivin}

\section{Discussion}

Human mesangioproliferative glomerulonephritis (MsPGN), e.g. IgA nephropathy, is a kidney disease with high incidence [1]. Although complement system activation leading to the production of terminal C5b-9 complex has been found in the renal mesangium of MsPGN patients $[4,37,38]$, the relationship between C5b-9 and GMC lesion as well as the role and mechanism of C5b-9-mediated GMC injury remains unclear. Rat Thy-1N is a well-accepted animal model for studying MsPGN [7]. In order to solve above-mentioned problems of human MsPGN, we used the rat model of Thy-1N to perform a series of experiments. Reportedly, in the process of Thy- $1 \mathrm{~N}$ induction, rat GMCs showed different pathological changes such as cell apoptosis and proliferation as well as inflammatory cytokine secretion [10,11,30,31]. Our previous experiments have proved that the GMC injury in the rats with Thy- $1 \mathrm{~N}$ is sublytic C5b-9-dependent $[10,18,30]$. As for the sublytic C5b-9-induced GMC apoptosis, sublytic C5b-9 attack can activate pro-apoptotic signals, and increase the expression of numerous pro-apoptosis factors or molecules $[13,16,18]$. Because apoptotic GMCs are thought to potential contributor to the renal damage of Thy- $1 \mathrm{~N}$ rat, the role and mechanism of GMC apoptosis upon sublytic C5b-9 stimulation in Thy-1N rats needs to be further clarified.

It is well known that cell apoptosis in some nephritis is modulated by many regulatory factors e.g. transcription factors and transcriptional coregulators as well as miRNAs [9, $15,18,24,39]$. Our previous studies have demonstrated that the GMC apoptosis evoked by sublytic C5b-9 in rat Thy-1N is associated with the upregulation of some specific transcription factors (e.g. IRF-1) and transcriptional coregulators (e.g. p300) as well as the acetylation of transcription factors (e.g. IRF-1 acetylation by p300) $[15,18,20]$. Recently, several experiments have revealed that miR-200a and miR-1469 are involved in regulating cell apoptosis $[24,40]$. Given that miRNAs can affect target gene expression by binding to the complementary sites of mRNAs 3` UTR causing mRNA degradation or translation inhibition $[21,23]$, and many researchers have found that miRNAs such as miR-148b, miR-29c and miR-29b are important regulators of viral infection, cancer or diabetes including MsPGN $[24-28,39,41]$, the effects of miRNAs on the GMC apoptosis mediated by sublytic C5b-9 in Thy-1N rats and the underlying mechanism needs to be explored.

In the present study, rat Thy-1N model was firstly established, with the sublytic C5b-9 complex on rat GMC membrane assembled, then the changes in miRNA expression profile both in the renal tissues of Thy-1N rats (in vivo) and in the GMCs stimulated by sublytic C5b-9 (in vitro) were detected and analyzed through miRNA microarray. The data revealed that a total of 17 miRNAs increased simultaneously both in vivo and in vitro, 11 of which were further validated by real-time PCR as being upregulated. In order to identify the GMC apoptosis-related miRNA, these 11 miRNAs were overexpressed in rat GMCs, and the results demonstrated that miR-150-3p, miR-328a-5p, miR-409-3p, miR-3546 and miR-3584$5 p$ markedly increased the expression of cleaved caspase 3 (a marker of cell apoptosis), especially miR-3546. Besides, our data showed a marked elevation of miR-3546 level both in the renal tissue of Thy- $1 \mathrm{~N}$ rats (peaked at $2 \mathrm{~h}$ in vivo) and in the GMCs stimulated with sublytic C5b-9 (peaked at $3 \mathrm{~h}$ in vitro). Furthermore, increase of miR-3546 activity by mimic or decrease of miR-3546 activity by inhibitor in the GMCs could significantly increase or decrease the number of apoptotic GMCs upon sublytic C5b-9 incubation. Based on these results, miR-3546 was selected to further determine its effect on sublytic C5b-9-triggered GMC apoptosis and the related regulatory mechanism. Notably, miR-3546 belongs to miR208 family, and though several documents have pointed out that miR-208 has regulatory role in myocardial injury [42-44], there has not been any report about the function of miR-3546.

As is mentioned above, miRNAs can bind to the complementary sites in the 3'UTR of mRNAs, leading to mRNA degradation or translation inhibition, and ultimately regulating target gene expression $[21,23]$. To further study the role of miR-3546 in sublytic C5b-9induced GMC apoptosis, we used several bioinformatics tools to predict putative target gene of miR-3546. Since it has been reported that SOX4 can promote cell proliferation and inhibit cell apoptosis in diabetes and many cancers [45-47], the detection of SOX4 expression both 
in vivo and in vitro were then carried out and the results exhibited that SOX4 expression was reduced remarkably. Meantime, the SOX4 expression in the GMCs could be greatly suppressed or enhanced by the mimic or inhibitor of miR-3546 respectively. In addition, overexpression or knockdown of SOX4 gene could impede or facilitate GMC apoptosis triggered by sublytic C5b-9, and SOX4 overexpression could significantly reverse the GMC apoptosis mediated by miR-3546 mimic. Mechanically, miR-3546 could bind to the 3`UTR of SOX4 mRNA and markedly inhibit its activity. Collectively, these data indicate that miR-3546 in rat GMCs can be upregulated by sublytic C5b-9 stimulation and enhanced GMC apoptosis via directly targeting SOX4. Here, it is worth mentioning that Pramoonjago P et al. [48] have reported that SOX4 repression could cause ACC3 cells apoptosis. Similarly, our present data also proved an anti-apoptotic function of SOX4 in rat GMCs with the attack of sublytic C5b-9.

A recent study has shown that SOX4 inhibition could induce melanoma cell apoptosis via p65 downregulation [49]. Given that SOX4 can be inhibited by miR-3546 in sublytic C5b9-induced GMC apoptosis, we hypothesized that downstream to SOX4 is some anti-apoptosis molecules which expression level might be downregulated in our experimental model as well. To test our hypothesis, we focused on the inhibitor of apoptosis gene (IAP) family members such as survivin, because several studies have confirmed that survivin is capable of regulating cell apoptosis [50-52], e.g. sodium arsenite can depress survivin expression, leading to cell growth inhibition and adult T-cell leukemia (ATL) cells apoptosis [53]. The subsequent experiments proved that the level of survivin was decreased both in vivo and in vitro, and the downregulation phase of survivin expression was similar to that of SOX4. Moreover, the overexpression or knockdown of SOX4 gene could significantly enhance or suppress the survivin synthesis in rat GMCs separately. Notably, miR-3546 could indirectly affect survivin expression via SOX4, and SOX4 overexpression could rescue the survivin repression mediated by miR-3546 mimic. Furthermore, luciferase reporter assay showed that SOX4 overexpression significantly augmented the activity of survivin promoter in the GMCs and HEK-293T cells, and ChIP assay revealed that SOX4 could bind to the region $(-1,278$ to $-853 \mathrm{nt}$ ) of survivin promoter. Besides, the expression level of survivin gene could affect sublytic C5b-9-induced GMC apoptosis, and the survivin overexpression greatly obstructed the GMC apoptosis mediated by miR-3546 mimic. Together, these findings implicate that the upregulation of miR-3546 and downregulation of SOX4 and survivin expression can really affect the GMC apoptosis triggered by sublytic $\mathrm{C} 5 \mathrm{~b}-9$. Because the experiments were performed in vitro, the effects of miR-3546, SOX4 and survivin on regulation GMC apoptosis in vivo requires further investigated.

\section{Conclusion}

In summary, our current study demonstrated that miRNA profiles were changed both in the renal tissues of Thy-1N rat (in vivo) and in the GMCs stimulated with sublytic C5b-9 (in vitro), and the increase of miR-3546 expression was followed by the decrease of SOX4 and survivin both in vivo and in vitro. The upregulated miR-3546 not only enhanced GMC apoptosis, but also reduced the synthesis of SOX4 and survivin. The mechanism of SOX4 and survivin downregulation in the GMCs treated by sublytic C5b-9 was associated with direct binding of miR-3546 to SOX4 3'UTR as well as the binding of SOX4 to the region -1, 278 to -853 nt on survivin promoter, which was identified for the first time. Collectively, these data implicate that miR-3546/SOX4/survivin axis plays a promoting role in the GMC apoptosis induced by sublytic C5b-9 in rat Thy-1N. Our findings not only provide a new insight into the pathogenesis of rat Thy-1N and human MsPGN, but also supply some potential targets for treating MsPGN. 


\section{Cellular Physiology Cell Physiol Biochem 2018;49:1898-1917 \begin{tabular}{ll|l} 
and BiOChemistry & DOI: 10.1159/000493652 & $\begin{array}{l}\text { C } 2018 \text { The Author(s). Published by S. Karger AG, Basel } \\
\text { www.karger.com/cpb }\end{array}$ \\
\hline
\end{tabular}

\section{Acknowledgements}

The study was supported by the grants from National Natural Science Foundations of China (81471626, 31470853 and 31500701).

\section{Disclosure Statement}

The authors declare that no conflicts of interest exist.

\section{References}

1 Lai KN: Pathogenesis of IgA nephropathy. Nat Rev Nephrol 2012;8:275-283.

2 Tumlin JA, Madaio MP, Hennigar R: Idiopathic IgA nephropathy: pathogenesis, histopathology, and therapeutic options. Clin J Am Soc Nephrol 2007;2:1054-1061.

- Floege J, Amann K: Primary glomerulonephritides. Lancet 2016;387:2036-2048.

4 Miyamoto H, Yoshioka K, Takemura T, Akano N, Maki S: Immunohistochemical study of the membrane attack complex of complement in IgA nephropathy. Virchows Arch A Pathol Anat Histopathol 1988;413:7786.

5 Nasri H, Ahmadi A, Rafieian-Kopaei M, Bashardoust B, Nasri P, Mubarak M: Association of glomerular C4d deposition with various demographic data in IgA nephropathy patients; a preliminary study. J Nephropathol 2015;4:19-23.

-6 Rosenblad T, Rebetz J, Johansson M, Bekassy Z, Sartz L, Karpman D: Eculizumab treatment for rescue of renal function in IgA nephropathy. Pediatr Nephrol 2014;29:2225-2228.

-7 Yamamoto T, Wilson CB: Complement dependence of antibody-induced mesangial cell injury in the rat. J Immunol 1987;138:3758-3765.

8 Sadlier DM, Ouyang X, McMahon B, Mu W, Ohashi R, Rodgers K, Murray D, Nakagawa T, Godson C, Doran P, Brady HR, Johnson RJ: Microarray and bioinformatic detection of novel and established genes expressed in experimental anti-Thy1 nephritis. Kidney Int 2005;68:2542-2561.

-9 Liu L, Qiu W, Wang H, Li Y, Zhou J, Xia M, Shan K, Pang R, Zhou Y, Zhao D, Wang Y: Sublytic C5b-9 complexes induce apoptosis of glomerular mesangial cells in rats with Thy-1 nephritis through role of interferon regulatory factor-1-dependent caspase 8 activation. J Biol Chem 2012;287:16410-16423.

10 Zhang J, Li Y, Shan K, Wang L, Qiu W, Lu Y, Zhao D, Zhu G, He F, Wang Y: Sublytic C5b-9 induces IL-6 and TGFbeta1 production by glomerular mesangial cells in rat Thy-1 nephritis through p300-mediated C/EBPbeta acetylation. Faseb j 2014;28:1511-1525.

11 Yamamoto T, Wilson CB: Quantitative and qualitative studies of antibody-induced mesangial cell damage in the rat. Kidney Int 1987;32:514-525.

12 Gao L, Qiu W, Wang Y, Xu W, Xu J, Tong J: Sublytic complement C5b-9 complexes induce thrombospondin-1 production in rat glomerular mesangial cells via PI3-k/Akt: association with activation of latent transforming growth factor-beta1. Clin Exp Immunol 2006;144:326-334.

13 Qiu W, Che N, Feng X, Xia M, Wang H, Zhao D, Wang Y: Apoptosis of glomerular mesangial cells induced by sublytic C5b-9 complexes in rats with Thy-1 nephritis is dependent on Gadd45 gamma upregulation. Eur J Immunol 2009;39:3251-3266.

14 Jiang X, Zhang J, Xia M, Qiu W, Wang H, Zhao D, Wang Y: Role of activating transcription factor 3 (ATF3) in sublytic C5b-9-induced glomerular mesangial cell apoptosis. Cell Mol Immunol 2010;7:143-151.

15 Qiu W, Zhou J, Zhu G, Zhao D, He F, Zhang J, Lu Y, Yu T, Liu L, Wang Y: Sublytic C5b-9 triggers glomerular mesangial cell apoptosis via XAF1 gene activation mediated by p300-dependent IRF-1 acetylation. Cell Death Dis 2014;5:e1176.

16 Zhu G, Qiu W, Li Y, Zhao C, He F, Zhou M, Wang L, Zhao D, Lu Y, Zhang J, Liu Y, Yu T, Wang Y: Sublytic C5b-9 Induces Glomerular Mesangial Cell Apoptosis through the Cascade Pathway of MEKK2-p38 MAPK-IRF-1TRADD-Caspase 8 in Rat Thy-1 Nephritis. J Immunol 2017;198:1104-1118.

17 Lv Q, Yang F, Chen K, Zhang Y: Autophagy protects podocytes from sublytic complement induced injury. Exp Cell Res 2016;341:132-138. 


\section{Cellular Physiology Cell Physiol Biochem 2018;49:1898-1917

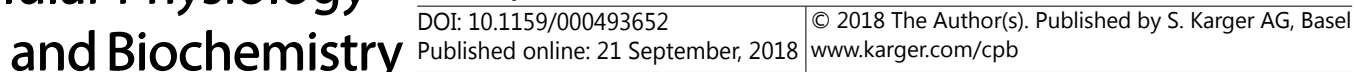

Yao et al.: Sublytic C5b-9 Induces GMC Apoptosis via miR-3546/SOX4/Survivin

18 He F, Zhou M, Yu T, Zhao D, Zhang J, Qiu W, Lu Y, Liu Y, Wang L, Wang Y: Sublytic C5b-9 triggers glomerular mesangial cell apoptosis in rat Thy-1 nephritis via Gadd45 activation mediated by Egr-1 and p300dependent ATF3 acetylation. J Mol Cell Biol 2016;8:477-491.

19 Lusthaus M, Mazkereth N, Donin N, Fishelson Z: Receptor-Interacting Protein Kinases 1 and 3, and Mixed Lineage Kinase Domain-Like Protein Are Activated by Sublytic Complement and Participate in Complement-Dependent Cytotoxicity. Front Immunol 2018;9:306.

-20 Xu K, Zhou Y, Qiu W, Liu X, Xia M, Liu L, Liu X, Zhao D, Wang Y: Activating transcription factor 3 (ATF3) promotes sublytic C5b-9-induced glomerular mesangial cells apoptosis through up-regulation of Gadd45alpha and KLF6 gene expression. Immunobiology 2011;216:871-881.

-21 Bartel DP: MicroRNAs: target recognition and regulatory functions. Cell 2009;136:215-233.

-22 Wang YC, Peterson SE, Loring JF: Protein post-translational modifications and regulation of pluripotency in human stem cells. Cell Res 2014;24:143-160.

23 Bartel DP: MicroRNAs: genomics, biogenesis, mechanism, and function. Cell 2004;116:281-297.

24 Fu H, Song W, Chen X, Guo T, Duan B, Wang X, Tang Y, Huang L, Zhang C: MiRNA-200a induce cell apoptosis in renal cell carcinoma by directly targeting SIRT1. Mol Cell Biochem 2018;437:143-152.

25 Sandbothe M, Buurman R, Reich N, Greiwe L, Vajen B, Gurlevik E, Schaffer V, Eilers M, Kuhnel F, Vaquero A, Longerich T, Roessler S, Schirmacher P, Manns MP, Illig T, Schlegelberger B, Skawran B: The microRNA-449 family inhibits TGF-beta-mediated liver cancer cell migration by targeting SOX4. J Hepatol 2017;66:10121021.

26 Moffett HF, Cartwright ANR, Kim HJ, Godec J, Pyrdol J, Aijo T, Martinez GJ, Rao A, Lu J, Golub TR, Cantor H, Sharpe AH, Novina CD, Wucherpfennig KW: The microRNA miR-31 inhibits CD8 $(+) \mathrm{T}$ cell function in chronic viral infection. Nat Immunol 2017;18:791-799.

-27 Satake E, Pezzolesi MG, Md Dom ZI, Smiles AM, Niewczas MA, Krolewski AS: Circulating miRNA Profiles Associated With Hyperglycemia in Patients With Type 1 Diabetes. Diabetes 2018;67:1013-1023.

28 Serino G, Pesce F, Sallustio F, De Palma G, Cox SN, Curci C, Zaza G, Lai KN, Leung JC, Tang SC, Papagianni A, Stangou M, Goumenos D, Gerolymos M, Takahashi K, Yuzawa Y, Maruyama S, Imai E, Schena FP: In a retrospective international study, circulating miR-148b and let- $7 \mathrm{~b}$ were found to be serum markers for detecting primary IgA nephropathy. Kidney Int 2016;89:683-692.

29 Ricklin D, Hajishengallis G, Yang K, Lambris JD: Complement: a key system for immune surveillance and homeostasis. Nat Immunol 2010;11:785-797.

30 Qiu W, Zhang Y, Liu X, Zhou J, Li Y, Zhou Y, Shan K, Xia M, Che N, Feng X, Zhao D, Wang Y: Sublytic C5b-9 complexes induce proliferative changes of glomerular mesangial cells in rat Thy-1 nephritis through TRAF6-mediated PI3K-dependent Akt1 activation. J Pathol 2012;226:619-632.

31 Wang Y, He Q Qin H, Xu J, Tong J, Gao L, Xu J: The complement C5b-9 complexes induced injury of glomerular mesangial cells in rats with Thy-1 nephritis by increasing nitric oxide synthesis. Life Sci 2006;79:182-192.

32 Couser WG, Pippin JW, Shankland SJ: Complement (C5b-9) induces DNA synthesis in rat mesangial cells in vitro. Kidney Int 2001;59:905-912.

-33 Cybulsky AV, Takano T, Papillon J, Bijian K, Guillemette J: Activation of the extracellular signal-regulated kinase by complement C5b-9. Am J Physiol Renal Physiol 2005;289:F593-603.

-34 Mi H, Guo N, Kejariwal A, Thomas PD: PANTHER version 6: protein sequence and function evolution data with expanded representation of biological pathways. Nucleic Acids Res 2007;35:D247-252.

35 Dy P, Penzo-Mendez A, Wang H, Pedraza CE, Macklin WB, Lefebvre V: The three SoxC proteins--Sox4, Sox11 and Sox12--exhibit overlapping expression patterns and molecular properties. Nucleic Acids Res 2008;36:3101-3117.

-36 Ambrosini G, Adida C, Altieri DC: A novel anti-apoptosis gene, survivin, expressed in cancer and lymphoma. Nat Med 1997;3:917-921.

-37 Rifai A, Chen A, Imai H: Complement activation in experimental IgA nephropathy: an antigen-mediated process. Kidney Int 1987;32:838-844.

-38 Coppo R: C4d deposits in IgA nephropathy: where does complement activation come from? Pediatr Nephrol 2017;32:1097-1101.

-39 Szeto CC, Li PK: MicroRNAs in IgA nephropathy. Nat Rev Nephrol 2014;10:249-256.

40 Xu C, Zhang L, Li H, Liu Z, Duan L, Lu C: MiRNA-1469 promotes lung cancer cells apoptosis through targeting STAT5a. Am J Cancer Res 2015;5:1180-1189. 


\section{Cellular Physiology Cell Physiol Biochem 2018;49:1898-1917

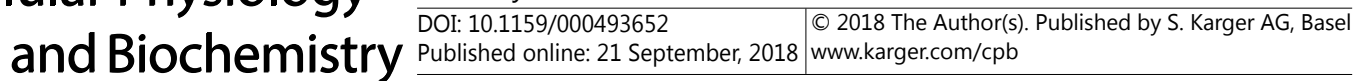 \\ Yao et al.: Sublytic C5b-9 Induces GMC Apoptosis via miR-3546/SOX4/Survivin}

41 Fang Y, Yu X, Liu Y, Kriegel AJ, Heng Y, Xu X, Liang M, Ding X: miR-29c is downregulated in renal interstitial fibrosis in humans and rats and restored by HIF-alpha activation. Am J Physiol Renal Physiol 2013;304:F1274-1282.

42 Kakimoto Y, Tanaka M, Kamiguchi H, Hayashi H, Ochiai E, Osawa M: MicroRNA deep sequencing reveals chamber-specific miR-208 family expression patterns in the human heart. Int J Cardiol 2016;211:43-48.

43 Nishimura Y, Kondo C, Morikawa Y, Tonomura Y, Torii M, Yamate J, Uehara T: Plasma miR-208 as a useful biomarker for drug-induced cardiotoxicity in rats. J Appl Toxicol 2015;35:173-180.

-44 Yan X, Liu J, Wu H, Liu Y, Zheng S, Zhang C, Yang C: Impact of miR-208 and its Target Gene Nemo-Like Kinase on the Protective Effect of Ginsenoside Rb1 in Hypoxia/Ischemia Injuried Cardiomyocytes. Cell Physiol Biochem 2016;39:1187-1195.

45 Chen ZZ, Huang L, Wu YH, Zhai WJ, Zhu PP, Gao YF: LncSox4 promotes the self-renewal of liver tumourinitiating cells through Stat3-mediated Sox4 expression. Nat Commun 2016;7:12598.

46 Xu EE, Sasaki S, Speckmann T, Nian C, Lynn FC: SOX4 Allows Facultative beta-Cell Proliferation Through Repression of Cdkn1a. Diabetes 2017;66:2213-2219.

-47 Pan W, Yu H, Zheng B, Gao Y, Li P, Huang Q Xie C, Ge X: Upregulation of MiR-369-3p suppresses cell migration and proliferation by targeting SOX4 in Hirschsprung's disease. J Pediatr Surg 2017;52:13631370.

48 Pramoonjago P, Baras AS, Moskaluk CA: Knockdown of Sox4 expression by RNAi induces apoptosis in ACC3 cells. Oncogene 2006;25:5626-5639.

49 Cheng Q, Du J, Xie L, Liu X, Li Z, Zuo F, Wu J, Xu J: Inhibition of SOX4 induces melanoma cell apoptosis via downregulation of NF-kappaB p65 signaling. Oncol Rep 2018;40:369-376.

50 Li PL, Zhang X, Wang LL, Du LT, Yang YM, Li J, Wang CX: MicroRNA-218 is a prognostic indicator in colorectal cancer and enhances 5-fluorouracil-induced apoptosis by targeting BIRC5. Carcinogenesis 2015;36:1484-1493.

51 Lamers F, van der Ploeg I, Schild L, Ebus ME, Koster J, Hansen BR, Koch T, Versteeg R, Caron HN, Molenaar JJ: Knockdown of survivin (BIRC5) causes apoptosis in neuroblastoma via mitotic catastrophe. Endocr Relat Cancer 2011;18:657-668.

52 Fan L, Sun G, Ma T, Zhong F, Wei W: Melatonin overcomes apoptosis resistance in human hepatocellular carcinoma by targeting survivin and XIAP. J Pineal Res 2013;55:174-183.

-53 Che XF, Zheng CL, Owatari S, Mutoh M, Gotanda T, Jeung HC, Furukawa T, Ikeda R, Yamamoto M, Haraguchi M, Arima N, Akiyama S: Overexpression of survivin in primary ATL cells and sodium arsenite induces apoptosis by down-regulating survivin expression in ATL cell lines. Blood 2006;107:4880-4887. 